Research Article

\title{
Investigation of Shock Initiation in Covered Charges under Shock Wave and Fragment Impacts
}

\author{
Xingwang Chen $(\mathbb{D}$, Jinxiang Wang $(\mathbb{D}$, Kui Tang $(\mathbb{D}$, Hongfei Wang $\mathbb{D}$, and Yuanbo Li $(\mathbb{D}$ \\ National Key Laboratory of Transient Physics, Nanjing University of Science and Technology, Nanjing 210094, China \\ Correspondence should be addressed to Jinxiang Wang; wjx@njust.edu.cn
}

Received 19 March 2021; Revised 5 May 2021; Accepted 31 May 2021; Published 8 June 2021

Academic Editor: Baoyu Ni

Copyright (c) 2021 Xingwang Chen et al. This is an open access article distributed under the Creative Commons Attribution License, which permits unrestricted use, distribution, and reproduction in any medium, provided the original work is properly cited.

\begin{abstract}
In the current work, a series of step-by-step research methods have been applied to address the damaging effects of near-field strong shock waves and high-speed fragments on covered charge. In the first step, the defects of covered plates due to high-speed fragments were simplified to penetrated notches, and then, these notches were used to evaluate the impact of shock wave loads on charges covered with metal plates. In the next step, we developed a theoretical model to take into account the shock initiation of charges covered with defected metal plates. Explosive initiation standards coupled with shock wave evolution characteristics were applied to specify the crucial conditions of explosive detonation. Finite element program, for instance, was applied for the simulation of shock initiation processes in pressed charges (when TNT was covered with a steel plate containing a penetrated notch), and then, numerical simulations were validated by experimental findings. Finally, the results obtained from the numerical simulations and theoretical model were applied to evaluate the impacts of shock wave intensity, the thickness of covered metal plate, and the geometrical features of penetrated notch on pressed charge shock initiation. The least squares method was applied to determine critical initiation criteria $(n$ and $\mathrm{K}$ ). Theoretical calculation results were found to be highly consistent with those obtained from numerical simulations, indicating that covered metal plates significantly contributed to charge protection. The results also revealed that notches could undermine the protective function of covered plates and the size and shape of notch significantly affected charge critical detonation distance. Critical detonation distances of noncontact explosions were found to be 25 and $81 \mathrm{~mm}$ for a $3 \mathrm{~mm}$ thick pressed TNT in the presence and absence of $45 \#$ steel-covered plate, respectively. According to the results, increase in the diameter of covered plates containing a cylindrical notch increased pressed TNT critical detonation distance. When dealing with a covered plate containing a normally reflected frustum notch, however, we figured out that any increase in normal reflection slope could decrease pressed TNT critical detonation distance.
\end{abstract}

\section{Introduction}

With recent progress in arms technology, safety of arms, particularly the safety of charges under external loads, has attracted great attention among researchers. Explosion shock waves and high-speed fragments are products of warhead charge detonation and shell fragmentation. In fact, the fragmentation effect of the explosion of conventional ammunition results from two main damage load factors $[1,2]$. Structural properties of covered plates also have significant effects on charge detonation. Taking this into account, researchers have studied the shock initiation of fragments and fragment groups with covered charge structures [3-6]. Walker and Wasley [7] applied the theory of thermal detonation to study the detonation and impact properties of PBX-9404 and other explosive materials. They concluded that $p^{2} t=C$, the initiation criterion of conventional heterogeneous explosives, could be applied to take the detonation and impact of insensitive ammunitions into account. Numerically simulating the sympathetic detonations of bare-bonded PBXN-109 explosive, Lu et al. [8] investigated the explosive shock wave effect on bare explosives. Howe et al. [9] used Euler software for numerical simulation of the sympathetic detonations based on the covered charge. In their study, they analyzed the explosion shock wave effect on acceptor charges at short distances of 
sympathetic detonation. They also tried to figure out how sympathetic detonation was affected by covered plate, donor explosive, sympathetic detonation distance, and shell thickness. Wang et al. [10] numerically simulated GHL explosive sympathetic detonations in steel shells to figure out how donor charge shell thickness could affect sympathetic distance. Using distributed experiment method, ChangMing [11] investigated charge explosive initiation under the combined effect of fragments and shock waves and analyzed fragmentation impact on explosive initiation taking explosive damage into account due to shock wave loading. The responses of defective metal plate-covered charges to super close field intensity shock waves from the perspective of ammunition safety have been studied previously. Therefore, evaluation of shock initiation due to explosive shock waves emitted from defective metal plate-covered charges (taking the safety design of ammunition into account) is essential.

In this study, pressed TNT (instance) and covered plate (45 \# steel) were applied for the investigation of covered charge shock initiation. A theoretical model was proposed to account for shock initiations in charges with defective steel plate covering. A numerical simulation method was applied for the analysis of the effect of shock initiation charges covered with defective steel plate subjected to near-field strong shock waves. Numerical simulation effectiveness was confirmed through some experiments, and theoretical calculations and numerical simulations were applied to evaluate the effects of covered plate initiation and various notch sizes. Distance effect on shock initiation was applied to specify significant initiation conditions.

\section{Theoretical Analysis}

Figure 1 shows the shock initiation model of defective metal-covered charge applied in the present study. This defective steel-covered charge consisted of donor and acceptor charges as well as a covered plate containing a penetrating notch. The radius of donor charge was denoted by $r$ and the covered plate had a thickness of $H$ and gradient of $\alpha$, respectively, which was in close contact with acceptor charge. Cylindrical and spherical charges had identical masses; detonation distance is $h$. Taking the two-dimensional plane assumption of a shock wave into account and ignoring sparse wave effect, we used the superposition and reflection principle of shock waves to analyze the evolution process of shock waves (shock wave propagation in air and shock wave superposition and reflection at covered plate notch).

2.1. Shock Wave Propagation. Baker's equation could be applied for measuring peak overpressure induced by air shock waves in the wake of an air explosion [12].

$$
\begin{aligned}
& \Delta P=\frac{2.036}{R}+\frac{0.194}{R^{2}}+\frac{0.004}{R^{3}}(0.05 \leq R \leq 0.5), \\
& \Delta P=\frac{0.067}{R}+\frac{0.301}{R^{2}}+\frac{0.431}{R^{3}}(0.5 \leq R \leq 70.9),
\end{aligned}
$$

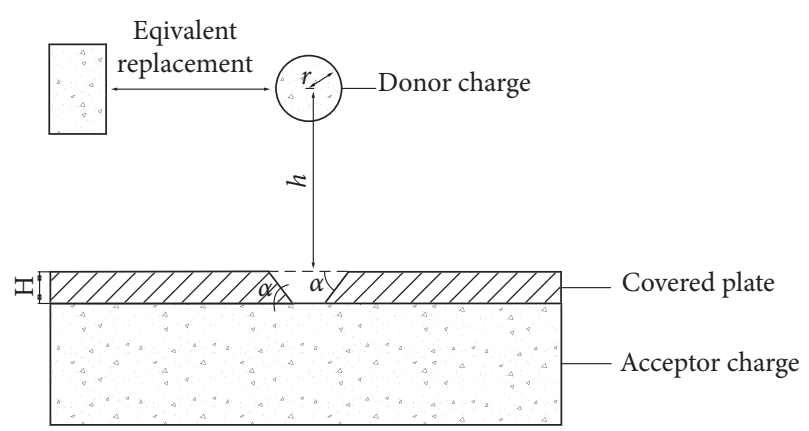

FIGURE 1: Schematic view of shock initiation in a charge with metal covering containing penetrated notch.

where $\Delta P$ represents the peak overpressure of shock wave and $R$ is the scale distance.

2.2. Shock Wave Reflection. A normal reflection phenomenon takes place when a normal shock wave reflection due to a detonation hits a solid wall, when air shock wave reflection created by point explosion hits the ground, or when two waves with comparable intensities collide in a condensed medium. On the basis of the shock pole curve and Hugoniot equation, we will have [12]

$$
\begin{gathered}
\tan \theta_{2}= \pm \frac{\sqrt{\left(p_{2}-p_{1}\right)\left(\left(1 / \rho_{1}\right)-\left(1 / \rho_{2}\right)\right)-\left(p_{2}-p_{1} / \rho_{1} q_{1}\right)^{2}}}{q_{1}-\left(p_{2}-p_{1} / \rho_{1} q_{1}\right)}, \\
e\left(p_{2}, \rho_{2}\right)-e\left(p_{2}, \rho_{2}\right)=\frac{1}{2}\left(p_{2}+p_{1}\right) \frac{1}{\rho_{1}}-\frac{1}{\rho_{2}},
\end{gathered}
$$

where $p_{2}, \rho_{2}$ denote the pressure and density applied by reflected wave and $\theta_{2}$ represents the angle between the frontal end of the reflected wave and solid wall. $p_{1}, \rho_{1}$ represent the pressure and density before reflection, respectively. Assuming air as an ideal gas, one could argue that the conditions required for the creation of normal reflections have been fulfilled.

$$
\left(\cot \alpha_{0}\right)^{2} \geq \frac{C_{1}^{2}-\left(D_{1}-u_{1}\right)^{2}}{D_{1}^{2}}=\left(\cot \alpha_{0}^{*}\right)^{2}
$$

where $\alpha_{0}$ is the angle between the frontal end of the incident wave and solid wall. $u_{1}, c_{1}, D_{1}$ are the fluid velocity, speed of sound, and shock wave velocity after the wave, respectively. Based on this equation, it could be concluded that normal reflection did not exist before the determination of $\alpha_{0}^{*}$. According to derivative equations of the shock wave, $\alpha_{0}<70^{\circ}$. This showed that normal reflection conditions were realized.

2.3. Shock Wave Superposition. The situation in which two shock waves with equal intensities collide is comparable to the situation when a shock wave is reflected after hitting a rigid wall. The equations applied for the calculation of reflection overpressure induced by a shock wave hitting a rigid wall could be presented as [12] 


$$
\Delta P_{d}=\Delta P\left[2+\frac{\cos \theta}{B}+\frac{(\gamma+1) \Delta P}{\gamma-1 \Delta P+2 \gamma P_{0}} \cos ^{2} \theta\right]
$$

where $\theta$ is the angle between the frontal ends of two shock waves and $B$ is obtained from the reflection parameter continuous condition when $\theta=\theta_{c r}$.

$$
B= \begin{cases}\cos \theta, & \theta \leq \theta_{c r} \\ \cos \theta_{c r}, & \theta \geq \theta_{c r}\end{cases}
$$

where $\theta_{c r}$ is the critical incident angle.

Since the impedance of airwaves is lower than those of baffle waves, compressed waves are reflected in air and transmitted to explosives. On the basis of Newton's third law and continuous interface condition, particles at both interface ends have identical velocity and exert equal pressure after reflection and transmission; therefore, one would have

$$
\begin{gathered}
P_{e}=T P_{d}, \\
n=\frac{\left(\rho_{0} c_{0}\right)_{1}}{\left(\rho_{0} c_{0}\right)_{2}} \\
T=\frac{2}{1+n},
\end{gathered}
$$

where $n$ denotes the acoustic impedance ratios of the two media, $T$ is the transmission coefficient, and $P_{e}$ is the pressure exerted on the surface of the explosive. Due to the much lower acoustic impedance of air than that of explosives, $n$ tended to be infinite. Therefore, it could be argued that the transmission coefficient $(T=2)$ was equal to that obtained in the wake of elastic wave reflection on the rigid wall.

\section{Numerical Simulation}

During the analysis of the shock initiation process in a defective metal plate-covered charge, we have applied AUTODYN-2D for the simulation of shock initiation in a metal plate-covered charge containing a penetrating notch. Several factors such as air domain, acceptor charge, defective covered plate, and donor charge were taken into account in the finite element calculation model, as shown in Figure 2. Both acceptor and donor charges were cylindrical pressed TNT, with a diameter and height of $35 \mathrm{~mm}$ and $30 \mathrm{~mm}$. Also, the thickness of the covered plate was $3 \mathrm{~mm}$ and the sizes of upper and lower levels of the notch were different. A pure Euler algorithm was applied to perform numerical calculations, the initiation point was considered to be at donor charge surface centre point, and the grid size is $1 \mathrm{~mm}$ divided into 10 segments. The specific material parameters of the covered plate and the TNT explosives are listed in Table 1 $[13,14]$. The parameters for the ignition and growth reactive model for acceptor charge are shown in Table 2 [14].

\section{Verification Test}

In order to analyze the effectiveness of numerical simulation, verification tests were used on covered charge shock initiation to obtain covered-pressed TNT sympathetic

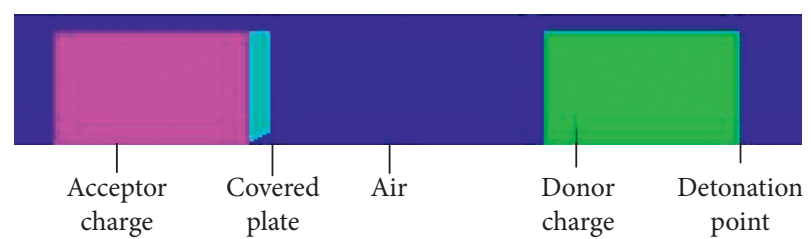

FIGURE 2: The model of the shock initiation with defective covered charge.

detonation distance during the noncontact explosion. Figure 3 shows shock initiation in covered charge and axisymmetric numerical calculation model. The test device consisted of acceptor charge, donor charge, booster, detonator, 45\# steel-covered plate, witness plate, and PPR sleeve. Acceptor charge directly contacted with $45 \#$ steel-covered plate and PPR sleeve was applied to detach $45 \#$ steel-covered plate and donor charge. The heights of the PPR sleeves applied in the four noncontact explosion tests are 15, 20,24, 26 , and $30 \mathrm{~mm}$ and the thickness of the covered plate is $3 \mathrm{~mm}$. The acceptor and donor charges used in this work were both cylindrical pressed TNT and the height and diameter of donor charge are $30 \mathrm{~mm}$ and $35 \mathrm{~mm}$, respectively. Also, the height and diameter of the acceptor charge are 15 and $50 \mathrm{~mm}$, respectively. The inner and outer diameters of the PPR sleeve are $33.7 \mathrm{~mm}$ and $40 \mathrm{~mm}$, respectively. Witness plate material was $45 \#$ steel with a diameter of $200 \mathrm{~mm}$ and a thickness of $100 \mathrm{~mm}$. Acceptor charge was placed in witness plate which was used to recover and judge the detonation of acceptor charge.

Table 3 shows the numerical simulation and experimental results obtained for the shock initiation of covered charges with different detonation distances. As given in Table 1, covered-pressed TNT sympathetic detonation distance in the noncontact explosion was in the range of $24-26 \mathrm{~mm}$ and critical sympathetic detonation distance determined through noncontact explosion numerical simulations was $24.5 \mathrm{~mm}$. The deviation between experimental and numerical simulation results is lower than $6.12 \%$. Therefore, it is concluded that numerical simulation results are highly consistent with test results.

Figure 4 shows the comparison chart of witness plate deformation for experimental and numerical simulation results at $20 \mathrm{~mm}$ intervals. After the acceptor charge was exploded, a slight depression was formed at the middle of the witness plate and a ring of pits was formed at its inner edge. The numerically calculated depth and diameter of the pit in the middle of the witness plate were $2.2 \mathrm{~mm}$ and $55.3 \mathrm{~mm}$, and corresponding experimental values were $2.0 \mathrm{~mm}$ and $54.2 \mathrm{~mm}$, respectively. The numerically calculated depth and diameter of the annular pit at the edge of the witness plate were $1.2 \mathrm{~mm}$ and $5.6 \mathrm{~mm}$ and the corresponding values obtained from experimental tests were $1.1 \mathrm{~mm}$ and $5.2 \mathrm{~mm}$, respectively. The deviation between numerical and experimental values obtained for the depth and diameter of the witness plate pit is no more than 3.49\% and $9.10 \%$, respectively. Therefore, it was found that numerical simulation results are agreed well with experimental results. 
TABLE 1: Parameters for TNT explosives and covered plate.

\begin{tabular}{lcccccccccc}
\hline \multirow{2}{*}{ Explosive } & Materials & $\rho_{0} / \mathrm{g} \cdot \mathrm{cm}^{-3}$ & $\mathrm{D}\left(\mathrm{m} \cdot \mathrm{s}^{-1}\right)$ & $P_{\mathrm{C}-\mathrm{J}} / \mathrm{GPa}$ & $\mathrm{A}(\mathrm{MBar})$ & $\mathrm{B}(\mathrm{MBar})$ & $R_{1}$ & $R_{2}$ & $\omega$ & $\mathrm{E}$ \\
& $\mathrm{TNT}$ & 1.60 & 6812 & 18.56 & 3.7 & 0.0323 & 4.15 & 0.95 & 0.3 & 0.07 \\
\hline \multirow{2}{*}{ Covered plate } & Materials & $\rho_{0} / \mathrm{g} \cdot \mathrm{cm}^{-3}$ & $\mathrm{G} / \mathrm{GPa}$ & $A / \mathrm{GPa}$ & $B / \mathrm{GPa}$ & $C$ & $n$ & $M$ & $T_{m} / K$ & $T_{r} / K$ \\
& $45 \#$ steel & 7.85 & 82.3 & 0.507 & 0.320 & 0.28 & 0.064 & 1.06 & 1765 & 298 \\
\hline
\end{tabular}

TABLE 2: Ignition and growth reactive flow model parameters for pressed TNT.

\begin{tabular}{|c|c|c|c|c|c|c|c|c|c|c|c|}
\hline$I / \mu \mathrm{s}^{-1}$ & $G_{1} / 100 \mathrm{GPa}$ & $\mathrm{G}_{2} / 100 \mathrm{GPa}$ & $a$ & $b$ & $c$ & $d$ & $e$ & $g$ & $x$ & $y$ & $\bar{z}$ \\
\hline $8 \bullet 10^{8}$ & 420 & 260 & 0.111 & 0.667 & 0.667 & 0.667 & 0.333 & 1 & 6 & 3 & 3 \\
\hline
\end{tabular}

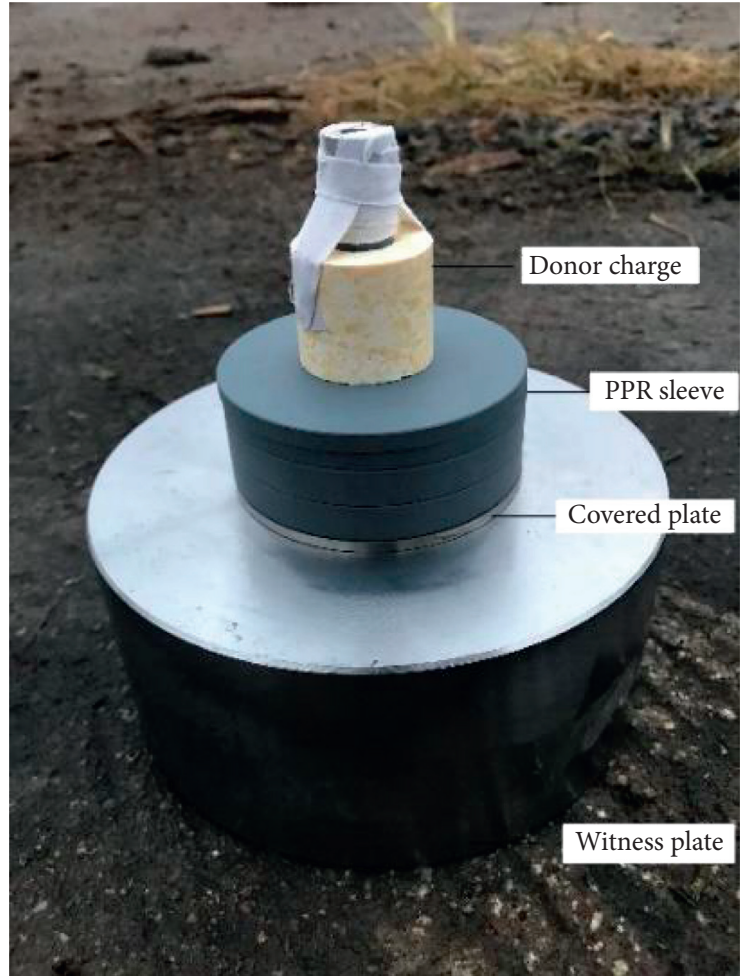

(a)

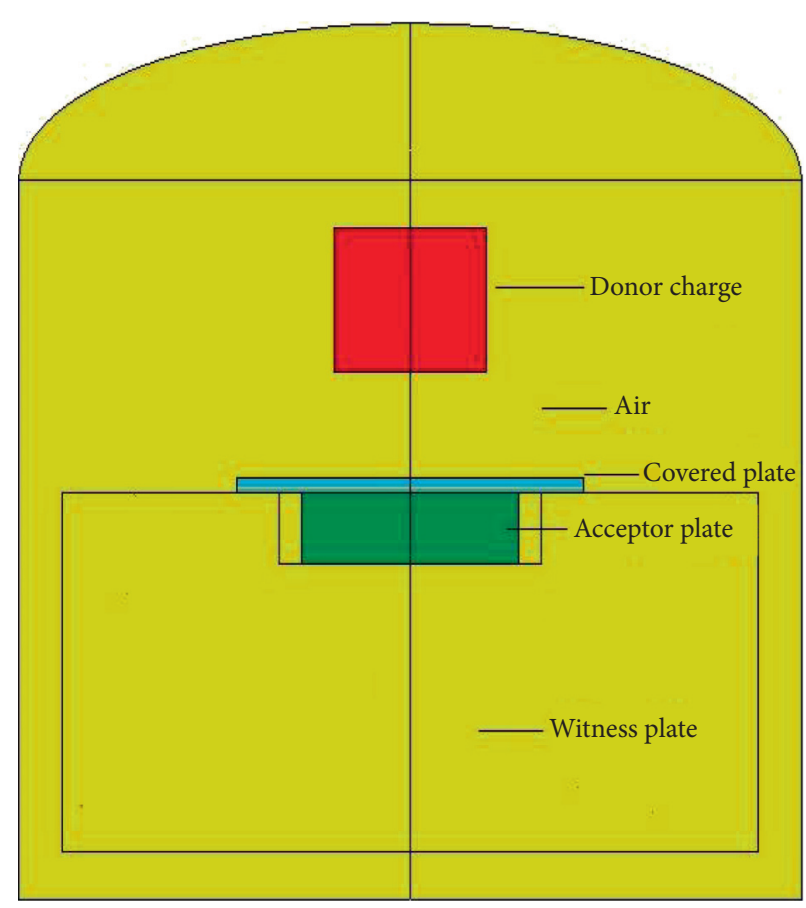

(b)

FIgURE 3: The photo of shock initiation on covered charge and numerical model.

TABLE 3: Simulation and experimental results obtained for covered charge shock initiation.

\begin{tabular}{|c|c|c|c|c|}
\hline & \multicolumn{2}{|r|}{ Test results } & \multicolumn{2}{|c|}{ Simulation results } \\
\hline & Sleeve height $(\mathrm{mm})$ & Explosion situation of acceptor charge & Sleeve height $(\mathrm{mm})$ & $\begin{array}{c}\text { Explosion situation of acceptor } \\
\text { charge }\end{array}$ \\
\hline Noncontact & 24 & Exploded & 24.5 & Exploded \\
\hline explosion & 26 & Unexploded & 25 & Unexploded \\
\hline
\end{tabular}

\section{Analysis and Discussion}

5.1. Comparison of Simulation and Theoretical Results. Gaussian point was used to measure pressure at various notch points at charge explosion moment. The values obtained from the abovementioned simulations and theoretical model are compared in Table 4. Assuming lower notch diameter to be fixed, researchers have changed the gradient of the covered plate through setting upper notch diameter and determined the effect of covered charge on detonations under six various conditions. Reflection and superposition pressures obtained from numerical simulations and theoretical calculations are compared below. The maximum variation between theoretical values and attenuation 


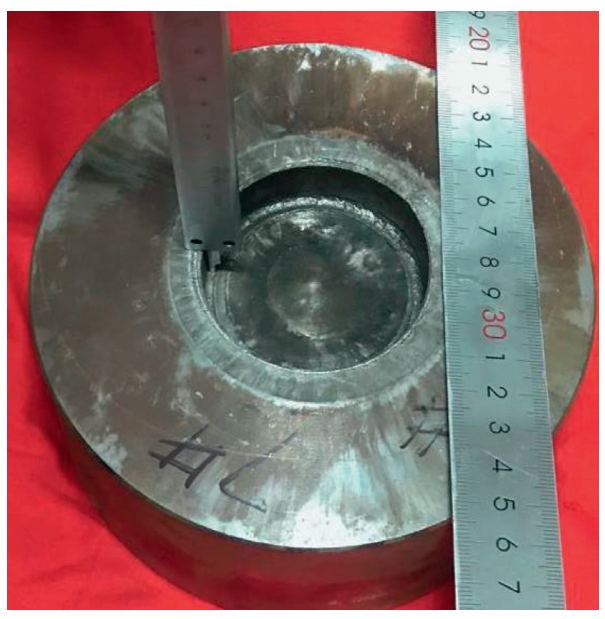

(a)

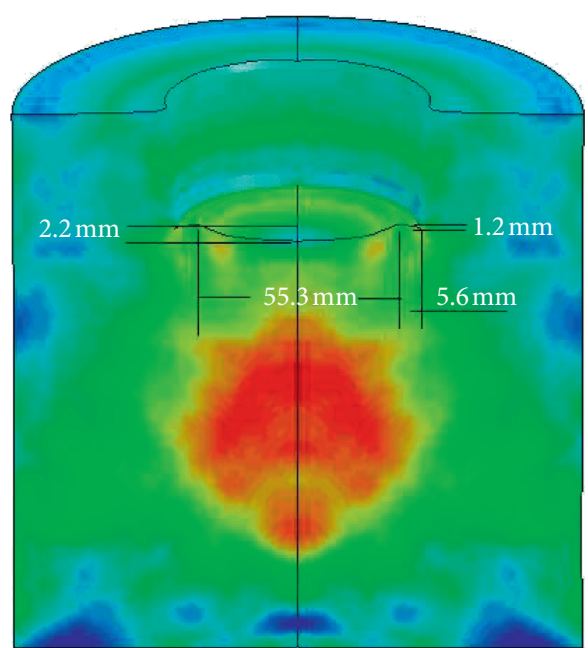

(b)

FIGURE 4: Comparison of witness plate deformation obtained from experimental tests and numerical simulations.

pressures was found to be $11.55 \%$, and that between theoretical values and reflection pressures was $7.45 \%$. Also, the maximum variation between theoretical values and superimposed shock wave pressures was $17.43 \%$. According to the findings, it can be concluded that theoretical values were consistent with simulation results.

Explosive detonation standard under strong shock wave loading could be presented as

$$
p^{n} \tau=K
$$

where $\tau$ is the time interval between detonation and shock waves, $p$ is the shock wave pressure at the explosive interface, $n$ is the empirical constant, and $K$ is an explosive-related parameter. The long-term sustained low-pressure shock wave can charge detonation only when the pressure of explosive interface $p$ is below critical initiation pressure $p_{c}$.

As the pressure exerted on the explosive charge was lower than the critical detonation pressure, the low-pressure shock waves could lead to detonation only when it was applied on explosive charge for a relatively long time. Therefore, the second form of shock initiation criterion $p^{2} \tau=k$ was applied. $n$ and $K$ are used to take into account charge detonation characteristics. According to the data presented in Table 2, $n=2.12$ and $K=1.24 e 12$ (International System of Units) were achieved by least squares method; it could be concluded that simplified theoretical model and shock initiation criterion obtained through fitting as well as acceptor explosive detonation characteristics when applying airborne shock wave can be adequately described up to a certain extent.

5.2. Shock Initiation Process Analysis. To investigate defective metal-covered charge shock initiation, we have analyzed the incomplete detonation and detonation growth processes of acceptor charge using a covered plate with lower and upper notch diameters of $1 \mathrm{~mm}$ and $5.5 \mathrm{~mm}$ and $4 \mathrm{~mm}$ and $1 \mathrm{~mm}$, respectively. Figure 5 shows typical explosion pressures at various acceptor charge times on shock initiation at lower and upper covered plate notch diameters of $1 \mathrm{~mm}$ and $5.5 \mathrm{~mm}$, respectively, and height of $42 \mathrm{~mm}$. According to Figure 5, the initial donor charge was detonated and the shock wave reached the covered plate notch surface at $t=13 \mu \mathrm{s}$. Moreover, the figure clearly shows that the superposition of shock wave occurred on acceptor charge upper surface and the covered plate pressure reached $586 \mathrm{MPa}$. Acceptor charge detonated once the shock wave reached critical initiation value $(5 \mu \mathrm{s})$ and the explosion created a high-pressure wave that rapidly spread to the explosive inner layer. Complete detonation occurred in $22 \mu \mathrm{s}$. In this case, the pressure of detonation reached 21.2 GPa. Figure 6 presents a typical explosion pressure cloud chart at various acceptor charge times on shock initiation at lower and upper covered plate notch diameters of $1 \mathrm{~mm}$ and $4 \mathrm{~mm}$, respectively, and height of $42 \mathrm{~mm}$. According to this figure, the shock wave hit the acceptor charge surface at $t=13 \mu \mathrm{s}$ and the covered plate notch was superimposed. Nevertheless, the covered plate pressure did not exceed $220 \mathrm{MPa}$. Within a few microseconds, charge pressure increased but its level was still below charge critical initiation energy. At $18 \mu \mathrm{s}$, the shock wave moved away from nonreflective boundaries and the acceptor charge was still intact.

Figure 7 (a) shows the distribution of gauge points, and the distance between adjacent Gauss points is $0.1 \mathrm{~cm}$. Complete and incomplete acceptor charge detonations for covered plate upper and lower notch diameters of $5.5 \mathrm{~mm}$ and $1 \mathrm{~mm}$ and $4 \mathrm{~mm}$ and $1 \mathrm{~mm}$ and height of $42 \mathrm{~mm}$ are presented in Figures $7(\mathrm{~b})$ and $7(\mathrm{c})$, respectively. Numbers 1-7 in Figure 7(a) represent the positions of acceptor charge in bottom-up order. Comparison of Figures 7(b) and 7(c) showed that the pressure exerted on acceptor charge surface reached $502 \mathrm{MPa}$ only when lower and upper covered plate notch diameters were 1 and $5.5 \mathrm{~mm}$, respectively. Explosive material instantaneously reacted to pressure increase and the maximum pressure of $3.8 \mathrm{GPa}$ was obtained. As detonation 


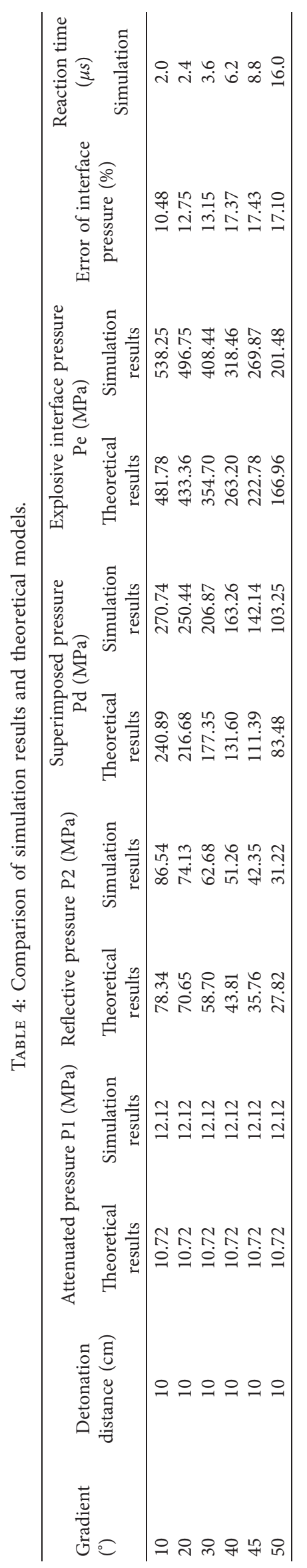




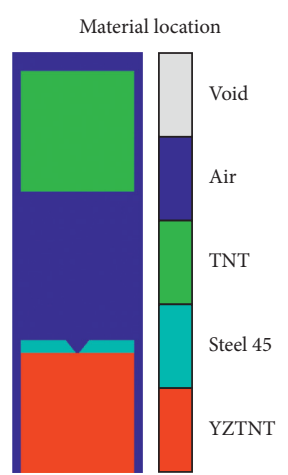

(a)

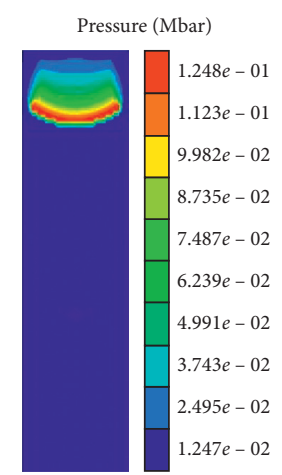

(b)

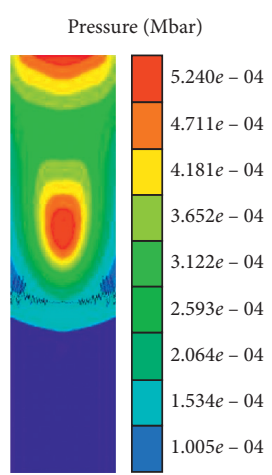

(c)

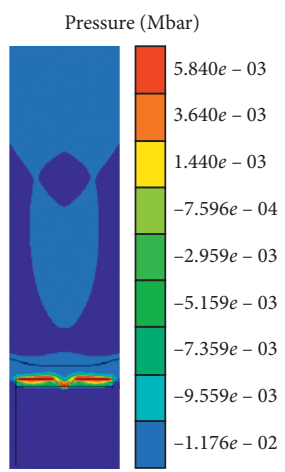

(d)

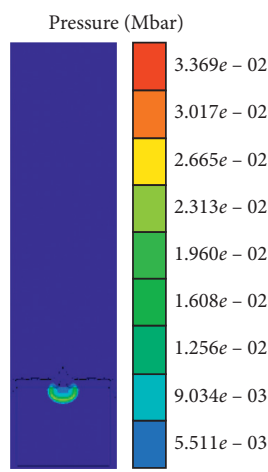

(e)

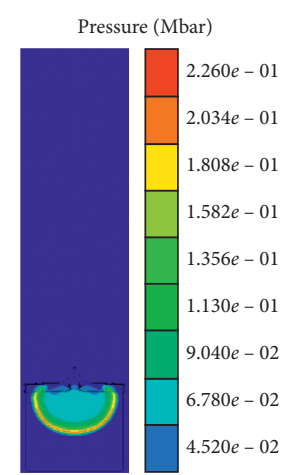

(f)

FIgURE 5: Acceptor charge cloud chart on shock initiation for lower and upper covered plate notch diameters of $1 \mathrm{~mm}$ and $5.5 \mathrm{~mm}$, respectively, and height of $42 \mathrm{~mm}$. (a) $t=0 \mu \mathrm{s}$. (b) $t=4 \mu \mathrm{s}$. (c) $t=10 \mu \mathrm{s}$. (d) $t=13 \mu \mathrm{s}$. (e) $t=18 \mu \mathrm{s}$. (f) $t=22 \mu \mathrm{s}$.

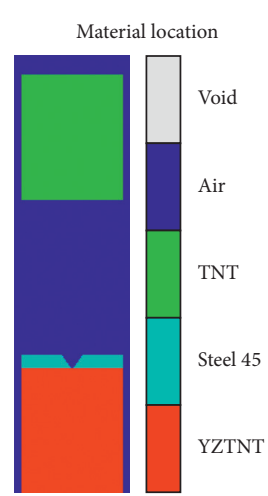

(a)

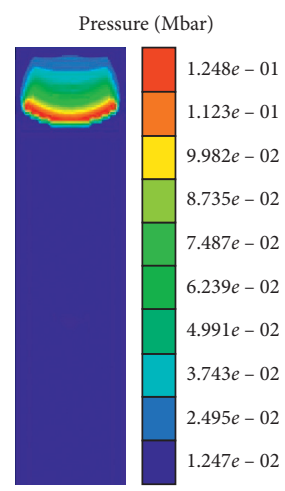

(b)

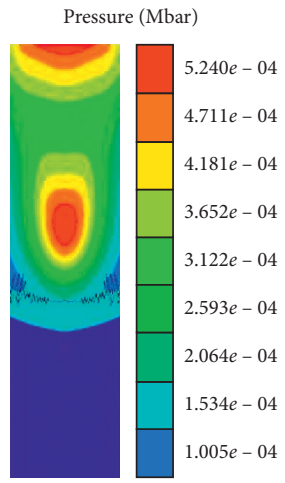

(c)

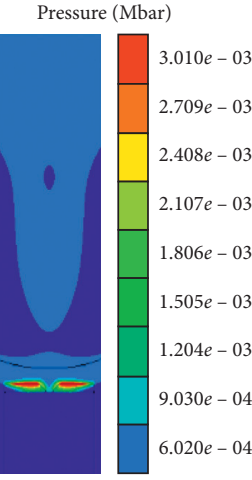

(d)

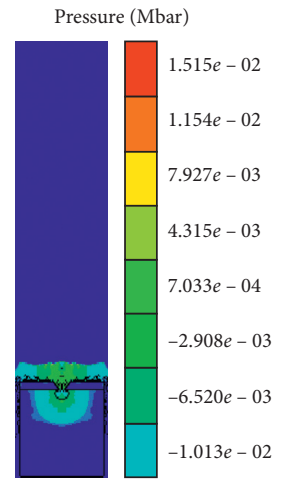

(e)

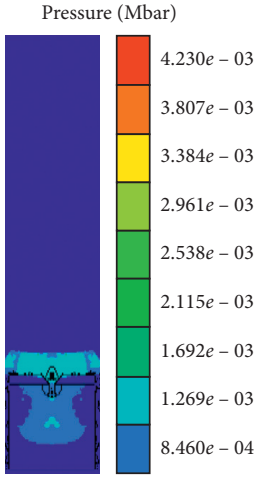

(f)

FIGURE 6: Acceptor charge cloud chart on shock initiation for lower and upper covered plate notch diameters of $1 \mathrm{~mm}$ and $4 \mathrm{~mm}$, respectively, and height of $42 \mathrm{~mm}$. (a) $t=0 \mu \mathrm{s}$. (b) $t=4 \mu \mathrm{s}$. (c) $t=10 \mu \mathrm{s}$. (d) $t=13 \mu \mathrm{s}$. (e) $t=18 \mu \mathrm{s}$. (f) $t=22 \mu \mathrm{s}$.

intensified, maximum pressure reached $22.2 \mathrm{GPa} 12 \mathrm{~mm}$ away from the acceptor charge surface and remained constant at a stable detonation state. At lower and upper covered plate notch diameters of $1 \mathrm{~mm}$ and $4 \mathrm{~mm}$, respectively, shock wave pressure applied on acceptor charge surface reached $220 \mathrm{MPa}$. A few microseconds later, the maximum pressure exerted on the acceptor charge reached 1.48 GP. Nevertheless, this pressure was not enough to incite acceptor charge into reaction. Moreover, once axial and lateral rarefaction waves were applied, the pressure exerted on the acceptor charge declined as propagation depth increased and the acceptor charge did not reach the explosion point.

5.3. Impact of Notch Size of Covered Plate. Investigation of the effect of covered plate notch size on the covered charge, by taking into account the vital role of covered plate upper and lower diameters in shock waves superposition and reflection, seems necessary. Due to the high accuracy of the abovementioned numerical simulation, we have numerically simulated defective metal-covered charge shock initiation by changing lower and lower sizes of the notch. Table 1 summarizes the results obtained from the simulation of sympathetic detonation distances of covered charges with various notch sizes. The critical detonation distances of noncontact explosions were found to be 25 and $81 \mathrm{~mm}$ for a $3 \mathrm{~mm}$ thick pressed TNT in the presence and absence of $45 \#$ steel-covered plate, respectively. According to the obtained results, increase in the diameter of covered plates containing cylindrical notches increased pressed TNT critical detonation distance. For covered plates with frustum notches, increase in normal reflection slope under normal reflection $\left(\alpha_{0}<70^{\circ}\right)$ decreased pressed TNT critical detonation distance.

Nonlinear least square was applied to quantitatively analyze covered plate notch size effect on detonation distance. As (8) shows, this method also enabled evaluating the relationship between notch size and detonation distance through fitting.

$$
h=b *\left(\alpha * \frac{\pi}{180}\right)+y
$$

where $\alpha$ is the notch slope, $h$ is the detonation distance, and $b$ and $y$ are the characteristic parameters of shock initiation which are related to the properties of the explosive and covered plate. By fitting the data presented in Table 5 $(b=-39.3 ; y=81.6)$, one can easily obtain the values. Figure 8 shows the relationship between notch slope and detonation distance. 

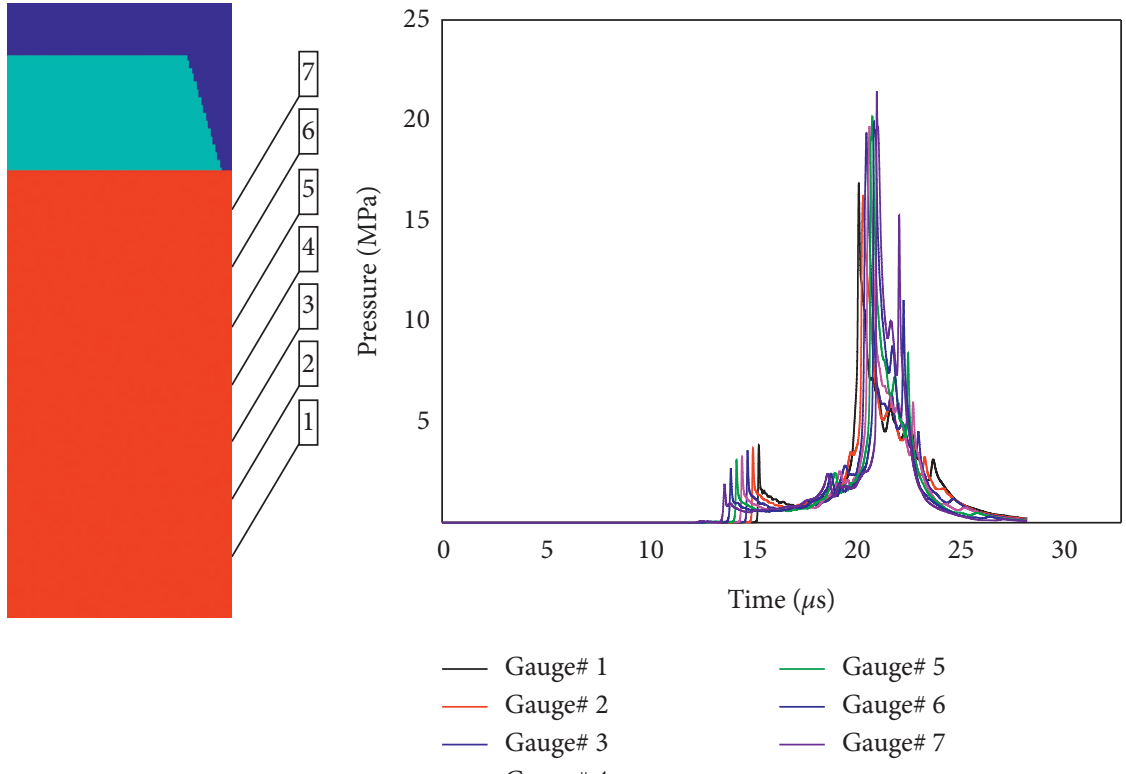

(a)

(b)

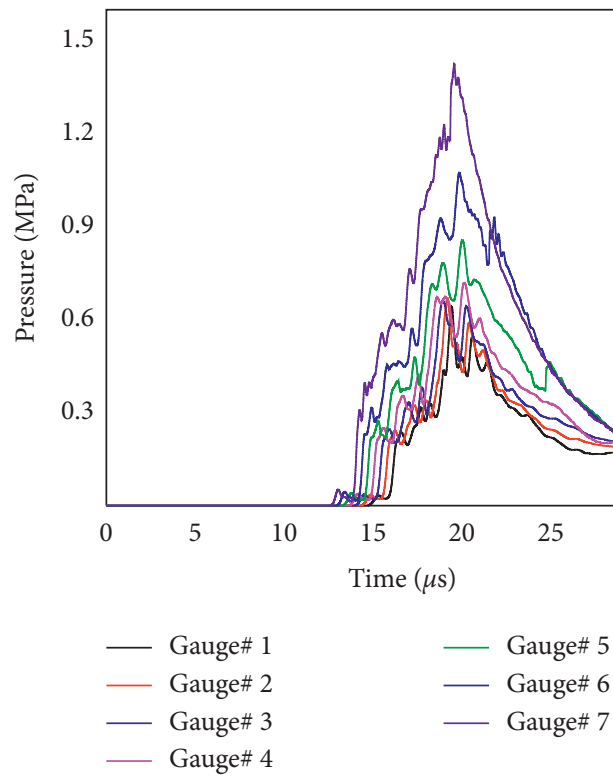

(c)

Figure 7: (a) Gauge point distribution. (b) The detonation development process. (c) Incomplete detonation process.

TABle 5: Simulation results obtained for detonation distance of covered charges with different notch sizes.

\begin{tabular}{lccccc}
\hline Serial number & $\begin{array}{c}\text { Thickness of the covered } \\
\text { plate }(\mathrm{mm})\end{array}$ & $\begin{array}{c}\text { Upper notch diameters } \\
(\mathrm{mm})\end{array}$ & $\begin{array}{c}\text { Lower notch diameters } \\
(\mathrm{mm})\end{array}$ & $\begin{array}{c}\text { Gradient }\left({ }^{\circ}\right) \\
\text { Detonation distance }(\mathrm{mm})\end{array}$ \\
\hline 1 & 0 & - & - & - & 81 \\
2 & 3 & 13 & 1 & 26.6 & 67 \\
3 & 3 & 10 & 1 & 33.7 & 59 \\
4 & 3 & 8.5 & 1 & 38.7 & 54 \\
5 & 3 & 7 & 1 & 53.1 & 53 \\
6 & 3 & 5.5 & 1 & 90 & 45 \\
7 & 3 & 4 & 1 & 90 & 38 \\
8 & 3 & 1 & 4 & 90 & 45 \\
9 & 3 & 4 & 7 & - & 53 \\
10 & 3 & 7 & 0 & & 25 \\
\hline
\end{tabular}




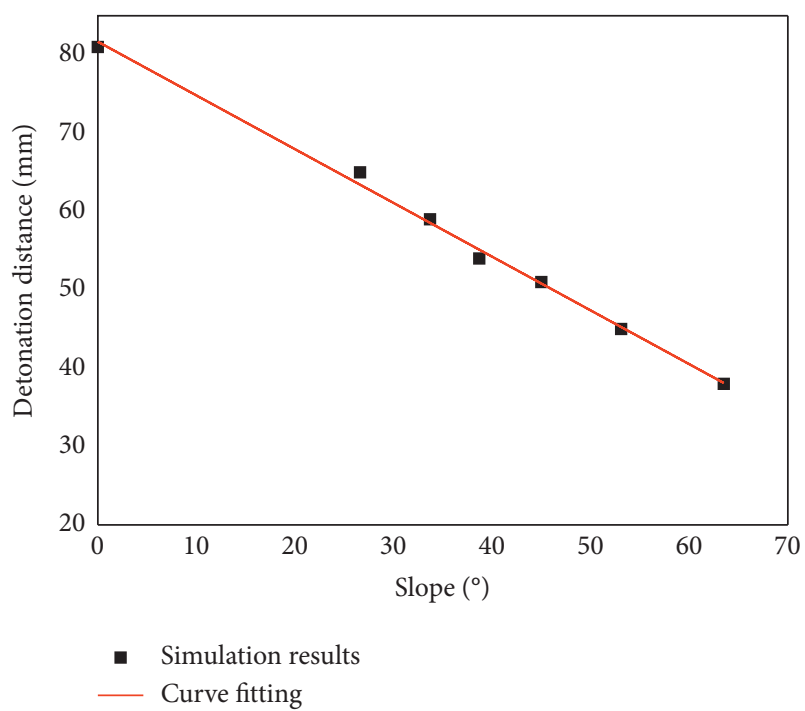

FIGURE 8: Relation between notch slope and detonation distance.

\section{Conclusions}

In the present study, attempts were made to formulate a theoretical model representing shock initiation in steelcovered charges with penetrated notches. Numerical simulation results were applied to determine the validity and verifiability of the developed theoretical calculation model. According to the obtained experimental results, the validity of the proposed numerical simulation was confirmed.

The following conclusions were drawn from this study:

(1) The results obtained from numerical simulations were consistent with experimental findings when comparisons were made based on the critical initiation distance and witness plate deformation. According to the obtained results, when the pure Euler algorithm was used, the calculation model could effectively take into account covered charge shock initiation.

(2) In this study, the least squares method was used for the determination of $n$ and $K$ (critical initiation criteria). The obtained results showed that, in the presence of near-field explosion shock wave, the theoretical model could more optimally account for covered charge detonation characteristics. Therefore, this model could be used as a theoretical reference for reliable investigations of shock initiations.

(3) The critical detonation distances of the noncontact explosion were found to be 25 and $81 \mathrm{~mm}$ for a $3 \mathrm{~mm}$ thick pressed TNT in the presence and absence of 45\# steel-covered plate, respectively. According to the obtained results, increase in the diameter of covered plates containing cylindrical notches increased pressed TNT critical detonation distance. For covered plates with frustum notches, increase in normal reflection slope under normal reflection $\left(\alpha_{0}<70^{\circ}\right)$ decreased pressed TNT critical detonation distance.

\section{Data Availability}

The data used to support the findings of this study are available from the corresponding author upon request.

\section{Conflicts of Interest}

The authors declare that they have no conflicts of interest.

\section{Acknowledgments}

This work was supported by the National Natural Science Foundation of China-NSFC (Grant no. 11672138).

\section{References}

[1] X. Song-lin, Y. Shi-qing, and Y. Li, "Safety standard system of ammunition and explosives," Safety and Environmental Engineering, vol. 19, pp. 85-89, 2012.

[2] Z. Rosenberg and E. Dekel, Terminal Ballistics, Springer Heidelberg, Dordrecht London New York, 2012.

[3] J. Chen, H. K. Ching, and F. Allahdadi, "Shock-induced detonation of high explosives by high velocity impact," Journal of Mechanics of Materials and Structures, vol. 2, no. 9, pp. 1701-1721, 2007.

[4] A. R. Gushanov, N. A. Volodina, G. V. Belov, V. N. Khvorostin, and D. M. Isheev, "Numerical simulation of experiments on the low-velocity impact on HMX-based he using explosive transformation initiation kinetics," Journal of Energetic Materials, vol. 28, no. 1, pp. 50-65, 2010.

[5] L. Qian, M. Qu, and G. Feng, "Study on terminal effects of dense fragment cluster impact on armor plate. part I: analytical model," International Journal of Impact Engineering, vol. 31, no. 6, pp. 755-767, 2005.

[6] L. Qian and M. Qu, "Study on terminal effects of dense fragment cluster impact on armor plate. part II: numerical simulations," International Journal of Impact Engineering, vol. 31, no. 6, pp. 769-780, 2005.

[7] F. E. Walker and R. J. Wasley, "Critical energy for shock initiation of heteropterous explosive," Explosive Stoffe, vol. 17, no. 1, pp. 9-13, 1969.

[8] P. M. Howe, Y. K. Huang, and A. L. Arbuckle, "A numerical study of detonation propagation between munitions," in Proceedings of the 7th Symposium International on Detonation, pp. 1055-1061, Annapolis, MD, USA, July 1982.

[9] J. P. Lu, I. J. Lochert, and D. L. Kennedy, "Simulation of sympathetic reaction rests for PBXN-109," in Proceedings of the insensitive Munitions \& Energetic Materials Technology Symposium, New York, USA, April 2006.

[10] C. Wang, J. Y. Wu, C. Lang et al., "Detonation tests and numerical simulations of shell explosives," Explosion and Shock Waves, vol. 30, pp. 152-158, 2010.

[11] O. Y. Chang-Ming, Z. P. Duan, B. P. Sun, Y. Liu, and F. L. Huang, "Experimental study on initiation of charge under combined shock wave and fragment impact," Explosion and Shock Waves, vol. 33, no. 1, pp. 63-66, 2013.

[12] J. H. Wang, Two-dimensional Unsteady Flow and Shock, Science Press, Beijing, China, 1994.

[13] G. Chen, C. Zhong-Fu, and J. L. Tao, "Johnson-cook failure model for 45 steel," in Proceedings of the Academic Conference of Chinese Society of Mechanics, Beijing, China, March 2005. 
[14] J. Guo-ping, "Two-dimensional shock initiation test and lagrange analysis of heterogeneous aggregate explosives," Master thesis, Hunan University, Hunan, China, 2005. 